\title{
Vehicle Tracking System using Android OS
}

\author{
Miss. Chetna Pardeshi ${ }^{1}$, Mr. Ashish Tulsankar ${ }^{1}$ \\ Government College of Engineering, Karad Shivaji University, Kolhapur ${ }^{1}$
}

\begin{abstract}
A vehicle tracking system is designed and implemented for tracking the movement of any vehicle from any location at any time. The proposed system made good use of a popular technology that combines a Smartphone application with a GPS. Global Positioning System (GPS) and Global system for mobile communication / General Packet Radio Service (GSM/GPRS) technology that is one of the most common ways for vehicle tracking. API of the Google Maps is used to display the vehicle on the map in the Smartphone application. Thus, users will be able to continuously monitor a moving vehicle on demand using the Smartphone application and determine the estimated distance and time for the vehicle to arrive at a given destination. In today's era everyone is using mobile phones for communication. At the same time Mobile Providers are also providing the variety of services to users. In attempt to expand on this, proposed a GPS based vehicle tracking android application for an organization to help to find addresses of their vehicles and locate their positions on mobile devices in real time adding some best services.
\end{abstract}

Index Terms: GPS; GPRS; Monitoring unit; Tracking unit.

\section{INTRODUCTION}

Global Positioning System (GPS) is a system composed of a network of 24 satellites of the United States, which are originally used in military services, and later allowed for commercial use. The satellites periodically emit radio signal of short pulses to GPS receivers. A GPS receiver receives the signal from at least three satellites to calculate distance and uses a triangulation technique to compute its two-dimension (latitude and longitude) position or at least four satellites to compute its three-dimension (latitude, longitude, and altitude) position. Once a location is computed, it can calculate an average speed and direction of traveling. Therefore, GPS is a key technology for giving device its position.

India has progressed on enormous rate that many companies have establish themselves here. These companies have a huge work force. Arranging the transportation to such huge force is difficult task. This transportation is arranged through local transport vehicles on yearly basis. But this has causes many mishaps like rape, burglary etc. Therefore, the proposed tracking system will help users in finding the location of vehicle through satellite communication. GPS and GSM based vehicle location and tracking system will provide effective, real time vehicle location, mapping and reporting this information back to monitoring device and improving the level of service provided. A GPS based vehicle tracking system informs user where the vehicle is and where it has been, how long it has been. The system uses geographic position and time information from the Global Positioning Satellites. Currently, mostly the existent tracking systems use techniques of virtual fence known as Geofence which compares the entity position with a predetermined zone or a point of interest, checking if the entity is inside or outside an area. Those techniques do not allow full coverage of the course, making difficult to determine if a truck or another delivery vehicle is travelling in a planned path.

Therefore, there is a need to use an alternative technique that allows continuous monitoring of travels, obtaining information of probable deviations or even emergency situations. This system consists of an android smartphone to continuously update location of vehicle with the help of drivers mobile. This device is called as tracking device which is continuously accessing its current location and is sending updates to monitoring device. At monitoring device which is an android application is providing the user with the exact location of the vehicles of his interest.

\section{RELATED WORK}

Yougui Liu, Baoxing Bai within the article "Research on GPRS Vehicle Location Network Service System" mentioned GPRS vehicle location network service system. The system is consisted of three major parts: GISnetwork workstations, network communications server and GPRS vehicle location terminal. First, GPRS vehicle location terminal was installed on the small car and connected to power supply, when the device was initialized; IP address of GPRS vehicle location terminal and port were set as fixed IP address of the server and GPRS vehicle location terminal communications service port. After setting, GPRS vehicle location automatically connected to GPRS vehicle location terminal communications services, and set IP address of GIS network workstations and port corresponding to the two communications links as IP address of network communications server and corresponding two service ports, operated login in, completed the connection of two communications links GIS network workstations [2]

Prof. Seema Vanjire, Umesh Kanchan, Ganesh Shitole, Pradnyesh Patil within the article "Location Based 
Services on Smart Phone through the Android Application" mentioned LBS components, LBS applications and some location collection services with the help of classes such as Location Manager Location Provider Geo-coding Google-Map [3]

Amol Dhumal, Amol Naikoji, Yutika Patwa, Manali Shilimkar, Prof. M. K. Nighot within the article "Vehicle Tracking System using GPS and Android OS" mentioned working of GPS and GLONASS for tracking the vehicle via android application. [4]

S Om Prakash, R Karthikaeyan within the article "Vehicle Tracking System with Smartphone Integration" mentioned working of vehicle tracking server, Client server architecture and various goals of previously implemented system with goals such as Individual task management, Safety tool, Administrative capabilities and its feasibility [5]

In Vehicle Tracking System for tracking the vehicle any tracking device is required. Now days, three navigation systems are available and people use those for tracking any object. The GNSS consist of three main satellite navigation systems. They are GPS (Global Positioning System), GLONASS and Galileo.

\section{PROPOSED METHODOLOGY}

System has three main modules transmitting unit, monitoring unit and server. Transmitting side performs tracking functionality. It tracks the vehicle through GPS and transmits its current location to the server. The main function of monitoring side is to providelogin interface to user and to show the google map with vehicle locations. Server woks as a central connector for transmitting unit and monitoring unit. As both transmitting side and monitoring side communicate with each other through Server only. If user is operating system for first time, then firstly he need to perform signup activity. While signing up user need to provide some basic information i.e. First name, Last name, Email Id etc. The necessary fields which user must fill while signing up. After sign up users all data gets store at server. The user name and password provided at a time of sign up is used for login to application.

As this application is made for an organizational so the all vehicles of organization must be registered. Registration of vehicle required organization name, vehicle no. and username. But for registering vehicle user must do login to the application. The login is done by providing username and vehicle number. Vehicle registration also requires username. This is because to provide more security to application user.

As shown in Fig. 1 mobile application communicates with server and access the remote database. Where at transmitting side Tracker application obtained its current location through GPS technology and update it to server.

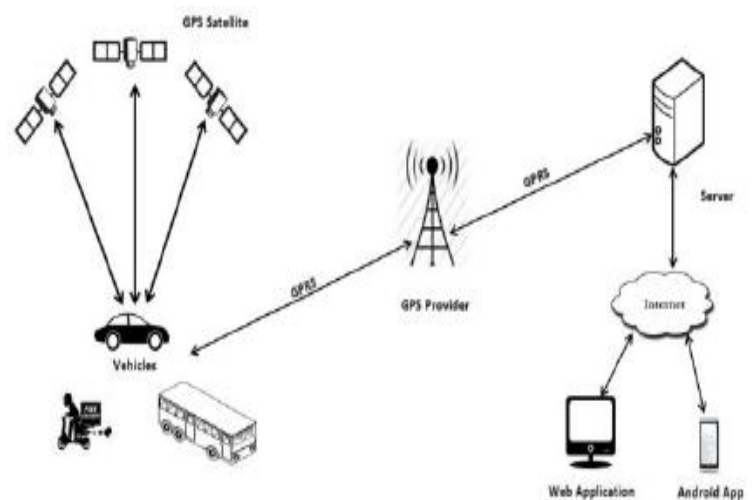

Fig 1.System Architecture

A. Project Components:

- GPS: GPS stands for Global Positioning System. The Global Positioning System (GPS) is a satellite radio navigation system allowing a GPS receiver to determine its position, velocity and time. Different types of positioning can be carried out using GPS receivers depending on the algorithms, type of measurements and corrections used in the navigation solution. GPS is a main module in this Vehicle tracking system as application has used it to get the exact location of respective vehicles. But to get exact location of any vehicle it need to be in a focus of four satellites.

- Monitoring unit: Monitoring unit is an Android Application through which Admin will get to know the actual position of proposed vehicles. This android provides the user interface through which user communicate with system. It provides login to the system. After login to the system user is will get google map with exact location of vehicles.

- Tracking Device: The tracking device will continuously request to the GPS satellite for its location information. At the same time GPS satellite will provide the location information to mobile devices.

In our system first android device will trap the signal from GPS receiver. GPS signals allow tracking devices to know its location, speed and mileage. The device communicates every minute or two via cellular internet network. The data is transmitted to our server within few seconds in order to provide real time alert status, location status and historical reports and fleet management.

\section{B. System Steps:}

When application is opened, the first page is Login or registration buttons. If the user is new to the system, he has to perform registration including indicating the role stating that Admin or Driver. And based on this, respective window will get opened for admin and driver. For Admin different window and for Driver different one.

And if the user is not new to the system, he will go for login. Login via Gmail account. After Login into system 
IARJSET

by using Username and Passcode authentication will get [3] complete and user will successfully enter into the system. According to the data entered at the time of registration the system will automatically redirect Admin and Driver to their respective path. That means if Admin then he will go to the page where he can see list of all the online drivers and if the person is user he will directly redirect to Google Map.

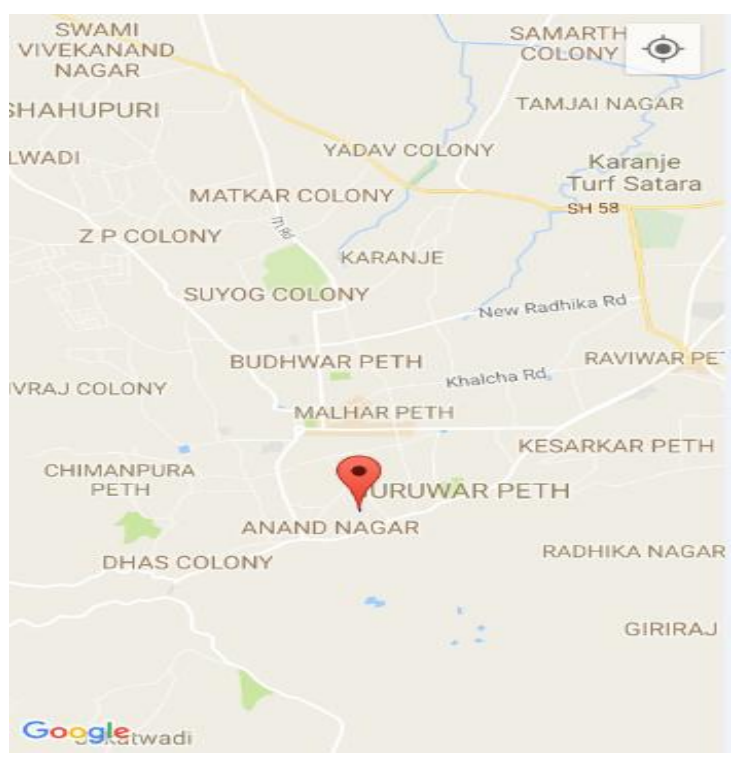

Fig 2. Location on Google Map

\section{CONCLUSION}

The proposed vehicle tracking android application allows organizations to track their vehicles and to get exact location of vehicle. The system allows those agencies to monitor the travelled routes through a android client that uses the Google Maps API and shows them on the map. The general evaluation result is that the system proved to be reliable as to view the positioning of the devices. If driver exceeds speed beyond safety limit, application will send the alert signal via android application to alert the driver. By using android application, admin can monitor the vehicle location, its speed and hereby uses various location based services for the best of society.

\section{ACKNOWLEDGMENT}

It is our privilege to express our sincerest regards to our project guide, Prof B.S. Yelure for their valuable inputs, encouragement, whole-hearted cooperation and constructive criticism throughout the duration of our project.

\section{REFERENCES}

[1] Changsheng Cai, Yang Gao "Precise Point Positioning Using Combined GPS and GLONASS Observations"

[2] Yougui Liu Baoxing Bai "Research on GPRS Vehicle Location Network Service System"
] Prof.Seema Vanjire, Unmesh Kanchan, Ganesh Shitole, Pradnyesh Patil "Location Based Services on Smart Phone through the Android Application"

[4] Amol Dhumal, Amol Naikoji, YutikaPatwa, Manali Shilimkar, Prof. M. K. Nighot "Vehicle Tracking System using GPS and Android OS"

[5] S Om Prakash, R Karthikaeyan "Vehicle Tracking System with Smartphone Integration"

[6] Hind Abdalsalam Abdallah Dafallah "Design and implementation of an accurate real time GPS tracking system" e-Technologies and Networks for Development (ICeND), 2014 Third International Conference on IEEE, April 29 2014-May 12014

[7] H. Stewart cobb "GPS pseudolites: theory, design, and applications", September 1997.

[8] SeokJu Lee; Girma Tewolde; Jaerock Kwon "Design and implementation of vehicle tracking system using GPS/GSM/GPRS technology and smartphone application" 2014 IEEE World Forum on, 6-8 March 2014. 